\title{
Research
}

\section{Clinical history for diagnosis of dementia in men:}

\author{
Caerphilly Prospective Study
}

\begin{abstract}
\section{Background}

Diagnosis of dementia often requires specialist referral and detailed, time-consuming assessments.

\section{Aim}

To investigate the utility of simple clinical items that non-specialist clinicians could use, in addition to routine practice, to diagnose all-cause dementia syndrome.

\section{Design and setting}

Cross-sectional diagnostic test accuracy study. Participants were identified from the electoral roll and general practice lists in Caerphilly and adjoining villages in South Wales, UK.
\end{abstract}

\section{Method}

Participants (1225 men aged 45-59 years) were screened for cognitive impairment using the Cambridge Cognitive Examination, CAMCOG, at phase 5 of the Caerphilly Prospective Study (CaPS). Index tests were a standardised clinical evaluation, neurological examination, and individual items on the Informant Questionnaire for Cognitive Disorders in the Elderly (IQCODE).

\section{Results}

Two-hundred and five men who screened positive $(68 \%)$ and $45(4.8 \%)$ who screened negative were seen, with 59 diagnosed with dementia. The model comprising problems with personal finance and planning had an area under the curve (AUC) of 0.92 (95\% confidence interval $[\mathrm{Cl}]=0.86$ to 0.97$)$, positive likelihood ratio (LR+) of $23.7(95 \% \mathrm{Cl}=5.88$ to 95.6), negative likelihood ratio (LR-) of 0.41 $(95 \% \mathrm{Cl}=0.27$ to 0.62$)$. The best single item for ruling out was no problems learning to use new gadgets (LR- of $0.22,95 \% \mathrm{Cl}=0.11$ to 0.43 ).

\section{Conclusion}

This study found that three simple questions have high utility for diagnosing dementia in men who are cognitively screened. If confirmed, this could lead to less burdensome assessment where clinical assessment suggests possible dementia.

\section{Keywords}

cohort studies; dementia; diagnostic tests: general practice; sensitivity and specificity.

\section{INTRODUCTION}

The diagnostic pathway for dementia, particularly in the UK, is changing. Casefinding seeks to identify people with possible dementia who have not been formally diagnosed, and has been a source of particular debate..$^{1-4}$ In contrast, a formal diagnosis aims to be as definitive as possible about the presence or absence of dementia, but the process of getting a diagnosis is often not patient-centred. ${ }^{5}$ In the general population there are often multiple contributing pathologies to dementia syndrome, ${ }^{6,7}$ with the association between Alzheimer's pathology and dementia weakening with age. ${ }^{8}$ Recent innovations in UK clinical practice have expanded the role of primary care in the diagnosis of dementia, such as GPs diagnosing patients without referral, ${ }^{9}$ or arranging for a specialist to visit primary care. ${ }^{10}$ In the UK, GPs are being encouraged to diagnose dementia independently, ${ }^{11}$ at least in people with moderately advanced disease. ${ }^{12}$

Few studies exist to provide an evidence-based approach to the diagnosis of dementia by GPs. The World Health Organization recommends that dementia is diagnosed by non-specialists in routine cases in low and middle income countries ${ }^{13}$; indeed the global clinical judgement of repeated GP consultations (three or four 10-minute-consultations) has moderate utility for the diagnosis of dementia with

S Creavin, MPhil, MRCP, NIHR academic clinical fellow in general practice; Y Ben-Shlomo, MSc, PhD, MRCP, professor, School of Social and Community Medicine, University of Bristol, Bristol. M Fish, MD, MRCP, consultant neurologist,

Department of Neurology, Musgrove Park

Hospital, Taunton. J Gallacher, PhD, professor, Department of Primary Care and Public Health, Cardiff University, Cardiff. A Bayer, FRCP, professor, Department of Primary Care and Public Health, Cardiff University, Academic Centre, University Hospital Llandough, Cardiff. an area under the curve (AUC) of $0.74 .^{14} \mathrm{An}$ AUC of 0.5 indicates a test no better than chance, such as coin-tossing, whereas an AUC of 1 indicates no diagnostic errors. In community-dwelling older people, a combination of functional activities questionnaire score ${ }^{15}$ with Mini Mental State Examination (MMSE) score ${ }^{16}$ and age had an AUC of 0.95. ${ }^{17}$ However, the MMSE is relatively time-consuming (between 7 and 18 minutes) ${ }^{18}$ and copyright-protected. GPs commonly report lack of time as a barrier to diagnosing dementia. ${ }^{19}$ It is desirable to identify the utility of simple clinical items that could be used easily by clinicians, as has been shown for the diagnosis of major depression in which two simple items have an AUC of $0.93 .{ }^{20}$

The present study used data from the Caerphilly Prospective Study (CaPS) to examine the utility of a variety of simple questions around everyday function, as well as conventional clinical assessment, for diagnosing dementia in older men. It aimed at identifying a quick and simple combination of questions that could be used by a GP as a further diagnostic test, in a person whom they suspected of having dementia after the usual clinical evaluation.

\section{METHOD}

\section{Participants}

CaPS is a cohort study of men that was established to investigate cardiovascular

\section{Address for correspondence}

Sam Creavin, School of Social and Community Medicine, University of Bristol, Canynge Hall, 39 Whatley Road, Bristol BS8 2PS, UK.

E-mail: sam.creavinabristol.ac.uk

Submitted: 12 November 2014; Editor's response: 17 December 2014, 2 March 2015; final acceptance: 16 March 2015.

\section{CBritish Journal of General Practice}

This is the full length article lpublished online $27 \mathrm{Jul}$ 2015) of an abridged version published in print. Cite this version as: $\mathbf{B r} \mathbf{J}$ Gen Pract 2015; DOI: 10.3399/bjgp15X686053 


\section{How this fits in}

Commissioners are interested in expanding the role of primary care in diagnosing some people with dementia without referral. However, GPs report lack of time as a barrier to this. The present study identifies three simple questions about functioning that had greater specificity than the composite MMSE (Mini Mental State Examination) for diagnosing dementia. These can be used easily by GPs as part of their assessment of a patient whom they believe may have a diagnosis of dementia.

disease. ${ }^{21}$ Men aged 45-59 years were identified from the electoral roll and general practice lists in Caerphilly and adjoining villages in South Wales, UK. Initial participation rate was $89 \%$ and 2512 men were examined in phase 1 (July 1979 to September 1983) and then followed-up at regular intervals. Cognition was assessed at phases 3 (November 1989 to September 1993), 4 (October 1993 to February 1997). and 5 (September 2002 to June 2004) using tests including the MMSE. ${ }^{16}$ At phase 5 all men who met screening criteria, ${ }^{22}$ as well as a sample of those who screened negative were invited for a clinical evaluation in their home or research clinic by a neurologist. Figure 1 shows the selection of participants and provides the screening criteria.

Figure 1. Flow of participation in the study.

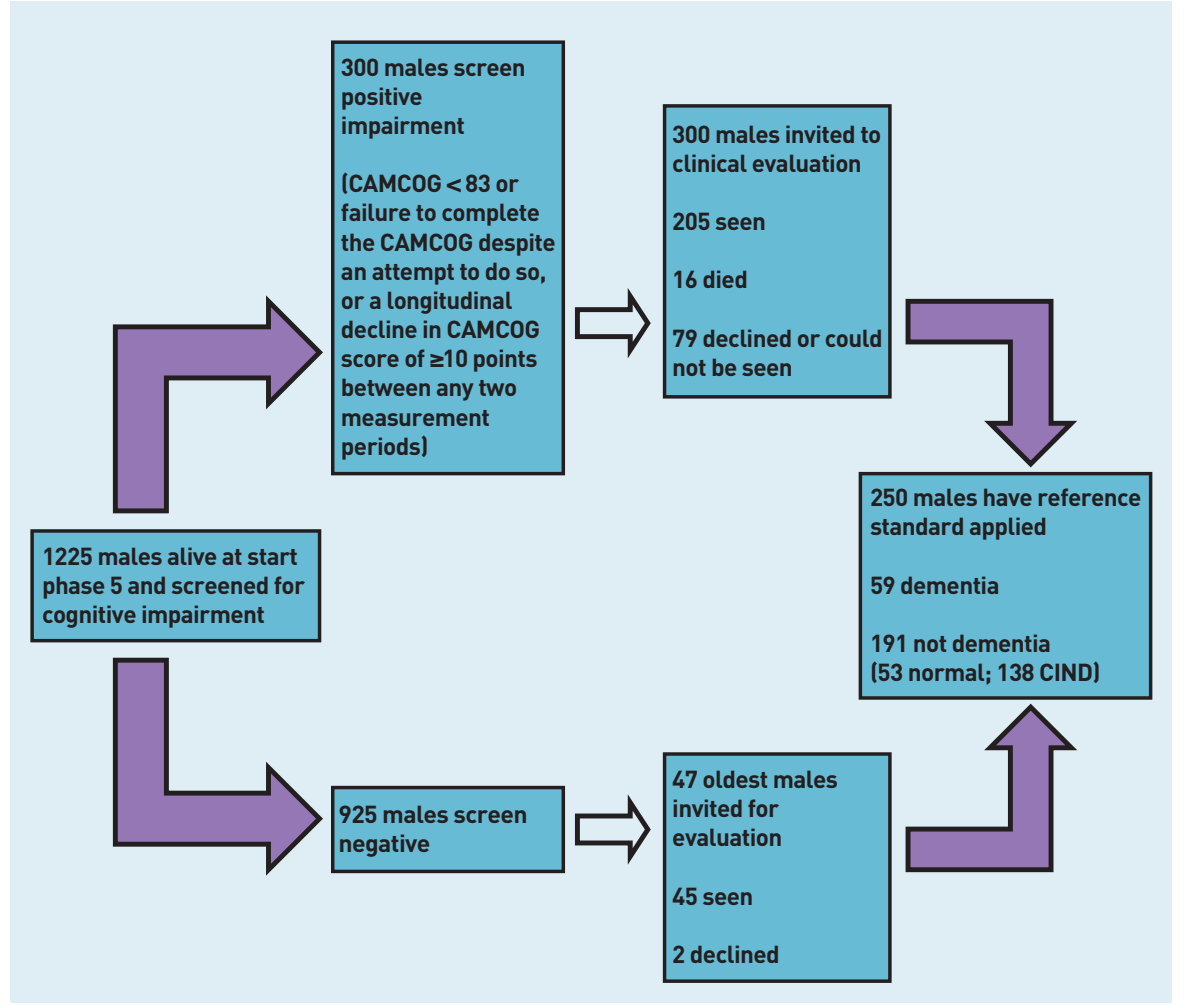

\section{Index test and reference standard}

The reference standard was a consensus diagnosis of dementia made by two clinicians with specialist training in memory disorders using the Diagnostic and Statistical Manual of Mental Disorders, Fourth Edition (DSMIV), ${ }^{23}$ after reviewing all relevant information including medical records lincluding investigations where available) and the clinical assessment. ${ }^{22}$

The index tests were items from the clinical assessment, incorporating a structured history and neurological examination (Cardiff modified CAMDEX ${ }^{24}$ and Frontal Assessment Battery ${ }^{25}$ ) and an informant interview (IQCODE $\left.{ }^{26}\right)$. Clinical assessment was conducted without prior knowledge of the patient and the informant questionnaire was conducted at the end of the evaluation. Individual items evaluated physical functioning (for example, incontinence), symptom patterns (for example, variability), personality (for example, aggression), and social functioning (for example, ability to plan), and were assessed either directly by the clinician or from the participant and informant (see Appendix 1 for detailed list of items).

\section{Statistical methods}

The diagnostic utility of each index item was assessed by calculating standard diagnostic measures, positive and negative predictive values (PPV, NPV), sensitivity, specificity, positive and negative likelihood ratio (LR+, LR-), diagnostic odds ratio (DOR), and AUC with $95 \%$ confidence intervals (Cls). As a result of small cell counts, any variable that had more than two categories (for example, normal versus abnormal) was dichotomised. Equivocal physical examination findings were analysed initially as present.

As it was desired to combine index items, only items that met conventional statistical significance $(P<0.05)$ were modelled, and where there were at least 20 subjects in each cell so diagnostic but infrequent items (for example, pout reflex) were excluded. Logistic regression models were used with dementia as the outcome and the index items as diagnostic predictors. ${ }^{27}$ First, backward stepwise logistic regression models were used with all items meeting the above criteria. Second, this was repeated but using only the five items with the greatest DOR and backwards and forwards stepwise models were run. This was also repeated using the Youden Index, ${ }^{28}$ as standard stepwise approaches maximise the AUC but not necessarily the diagnostic utility. ${ }^{28}$

For each model, the model probability of dementia was examined, and only models 


\section{Table 1. Cross-tabulation of cognitive impairment screen against} reference standard

\begin{tabular}{llllll} 
& & \multicolumn{3}{c}{ Dementia (DSM-IV) } & Notal \\
\cline { 3 - 5 } & & & Yes & No & Total \\
\hline Cognitive impairment screen $^{\text {a }}$ & Positive & 58 & 147 & 205 & \\
& & Negative & 1 & 44 & 45 \\
& & Total & 59 & 191 & 250
\end{tabular}

${ }^{2}$ Cambridge Cognitive Examination (CAMCOG) <83 or failure to complete the CAMCOG despite an attempt to do so, or a longitudinal decline in $C A M C O G$ score of $\geq 10$ points between any two measurement periods. in which the highest risk was at least $90 \%$ were selected, then goodness of fit statistics were calculated to choose between these models. ${ }^{29}$

In addition the following supplementary analyses were made: any subjects with a prior diagnosis of dementia were excluded; analyses were repeated but recoding equivocal clinical items as absent; imputation was used for missing item data using chained equations and creating seven simulated datasets. ${ }^{30}$ All analyses were conducted in Stata (version 13).

\section{RESULTS}

At the start of phase 5, 1225 men were alive and 300 were screen positive. Of those who were screen positive 205 (68\%) attended clinical assessment. In addition, 47 of the 925 men who screened negative were invited for clinical assessment, ${ }^{22}$ with 45 (95.7\%) of these subsequently assessed (see Figure 1 for details for non-response). Seven had already been given a diagnosis of dementia. The cognitive screening procedures were $98 \%$ sensitive for identifying men with dementia (Table 1).

Fifty-nine men $124 \% ; 95 \% \mathrm{Cl}=19 \%$ to $29 \%$ ) were diagnosed as having dementia (median age 77.8 years; interquartile range [IQR] 74.9-80.0) and $191(76 \% ; 95 \% \mathrm{Cl}=71 \%$ to $81 \%$ ) did not have dementia (median age 75.7 years; IQR 71.8-79.3). The median (IQR) MMSE score in men with dementia was 20 (16-23) compared with 25 (22-29) in those without dementia. One-hundred and thirty-eight men were identified as having cognitive impairment not dementia (CIND) and they were included in the no-dementia group for analysis.

Table 2 presents the sensitivity and specificity for the individual clinical items that were examined in combinations. The most sensitive items were participant report of any memory difficulty $191.1 \%$; $95 \%$ $\mathrm{Cl}=80.4 \%$ to $97.0 \%)$ or forgetting where things were left $191.1 \%, 95 \% \mathrm{Cl}=80.4 \%$ to $97.0 \%)$, but specificity was only $19.8 \%$ and
$31.9 \%$, respectively. Highly specific items were: informant report of problems with reasoning $(95.1 \% ; 95 \% \mathrm{Cl}=90.6 \%$ to $97.9 \%)$; hygiene $(95.3 \% ; 95 \% \mathrm{Cl}=90.9 \%$ to $98.0 \%)$; and disinhibited behaviour 194.7\%; 95\% $\mathrm{Cl}=89.9 \%$ to $97.7 \%$ ). A list of diagnostic utility of all single items, regardless of cell counts, is available from the authors on request; the diagnostic accuracy for some items could not be calculated because they were not identified in any men (details in Appendix 1).

The best performing individual items for $L R-, L R+$, and AUC were as follows: problems with 'learn new gadget' (LR- 0.22; $95 \% \mathrm{Cl}=0.11$ to 0.43$)$, problems with 'reasoning' (LR+ 10.4; $95 \% \mathrm{Cl}=5.06$ to 21.5), and problems with 'personal finance' (AUC 0.80; $95 \% \mathrm{Cl}=0.72$ to 0.88) (Table 3). The optimal cut-point for diagnosis of dementia using age alone was 75.8 years (LR- 0.61 ; $L R+1.40$; AUC 0.60). In contrast the utility of the MMSE at a traditional cut-point of $\geq 24$ indicating normality was LR- 0.28; LR+ 2.14; AUC 0.72 , and the utility of memory problem noted by informant or participant was LR0.45; LR+ 1.10; AUC 0.55 suggesting this alone is not a useful diagnostic feature in this population.

The results from the stepwise procedures using all or selected index items can be found in Table 4. There were 10 models meeting the criteria of predictive value and goodness of fit. All were better in terms of specificity than sensitivity and all but one had an AUC value $\geq 0.90$. It was judged that the best overall model comprised problems with personal finance and planning (AUC $0.92,95 \% \mathrm{Cl}=0.86$ to 0.97 ), but the model comprising personal finance and reasoning (AUC $0.86,95 \% \mathrm{Cl}=0.80$ to 0.93 ) had almost as good a performance profile based on a larger sample size.

Supplementary analyses when excluding the seven men who had an existing diagnosis of dementia, produced similar results for specificity, but sensitivity was lower (up to 20 percentage points). When analysing equivocal findings as normal the results were almost identical (to two decimal places) to the main analysis. Multiple imputation also resulted in similar results to the main analysis with specificity within $10 \%$ of the main estimates, but sensitivity often lower lup to 20 percentage points, details available from the authors). The AUC was consistent between imputed analyses and main.

\section{DISCUSSION}

\section{Summary}

This study found that combinations of 


\section{Table 2. Sensitivity and specificity for most useful individual clinical items}

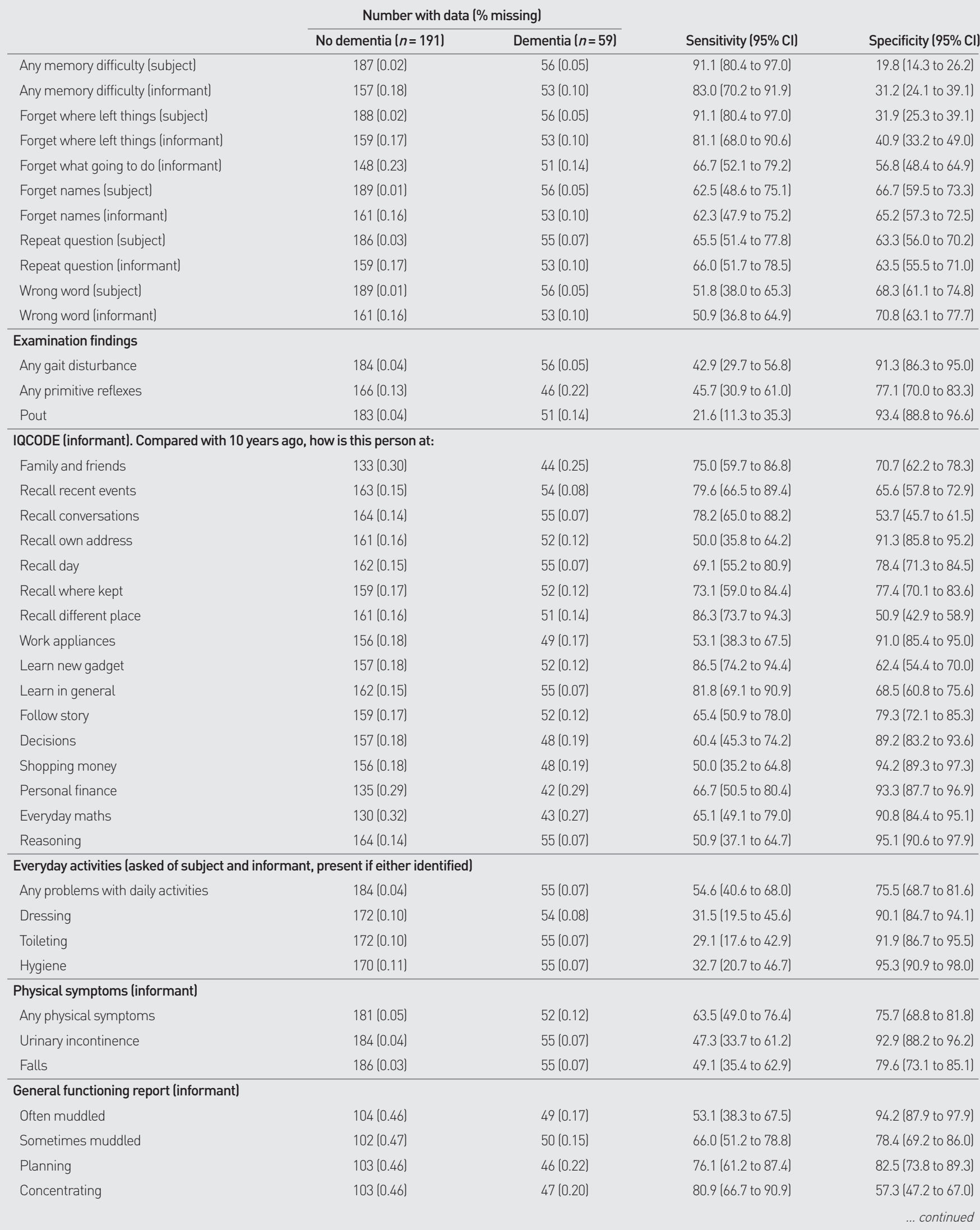


Table 2 continued. Sensitivity and specificity for most useful individual clinical items

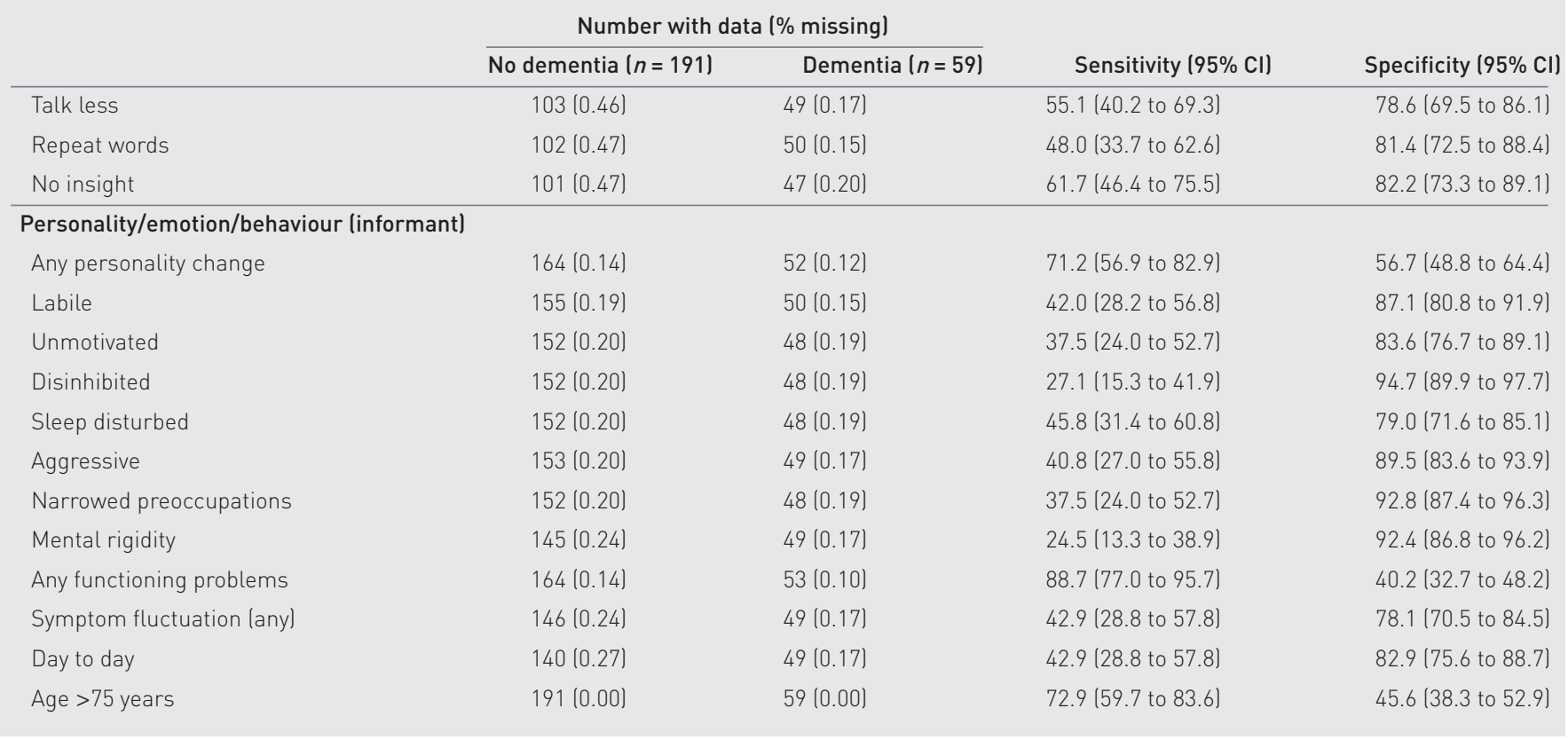

simple questions had comparable or better diagnostic utility than the MMSE, with high utility for ruling-in a diagnosis, in men who were cognitively screened. Abnormal gait and primitive reflexes were the most useful physical findings. Some individual items were highly specific (for example, reasoning), whereas others were very sensitive lany memory difficulty reported by subject), but a combined approach enhanced their value.

\section{Strengths and limitations}

CaPS was not designed as a diagnostic test accuracy study, but, for the present study, a sample was selected with a high prior probability of some cognitive impairment from a larger population-based sample, with a broad spectrum of disease. A recognised reference standard was used, applied in consensus by experienced clinicians and a wide range of questionnaire items as well as neurological assessment were examined. Several models were tested that combined the best items. The reference standard was subject to incorporation bias (or circularity ${ }^{31}$ ) as results of the index tests could have been used in reaching a final diagnosis, but this is a common problem with clinical definitions

\section{Table 3. Diagnostic utility of the best performing individual clinical items}

\begin{tabular}{lcccccc} 
Clinical item & $\mathbf{N}$ & LR+ $(95 \%$ CI) & LR- $(95 \%$ CI) & DOR (95\% CI) & AUC (95\% CI) & Youden (95\% CI) \\
\hline Reasoning & 219 & $10.4(5.06$ to 21.5$)$ & $0.52(0.39$ to 0.68$)$ & $20.2(8.45$ to 48.2$)$ & $0.73(0.66$ to 0.80$)$ & $0.46(0.31$ to 0.59$)$ \\
\hline Personal finance & 177 & $10.0(5.14$ to 19.5$)$ & $0.36(0.23$ to 0.55$)$ & $28.0(11.1$ to 70.3$)$ & $0.80(0.72$ to 0.88$)$ & $0.60(0.43$ to 0.73$)$ \\
\hline Often muddled & 153 & $9.20(4.05$ to 20.9$)$ & $0.50(0.37$ to 0.67$)$ & $18.5(6.95$ to 48.8$)$ & $0.74(0.66$ to 0.81$)$ & $0.47(0.31$ to 0.61$)$ \\
\hline Shopping money & 204 & $8.67(4.33$ to 17.4$)$ & $0.53(0.40$ to 0.71$)$ & $16.3(6.86$ to 38.8$)$ & $0.72(0.65$ to 0.79$)$ & $0.44(0.28$ to 0.58$)$ \\
\hline Everyday maths & 173 & $7.05(3.94$ to 12.6$)$ & $0.38(0.25$ to 0.58$)$ & $18.4(7.80$ to 43.2$)$ & $0.78(0.70$ to 0.86$)$ & $0.56(0.39$ to 0.69$)$ \\
\hline Learn new gadget & 209 & $2.30(1.83$ to 2.89$)$ & $0.22(0.11$ to 0.43$)$ & $10.7(4.60$ to 24.7$)$ & $0.74(0.68$ to 0.81$)$ & $0.49(0.36$ to 0.60$)$ \\
\hline Learn in general & 217 & $2.60(2.01$ to 3.37$)$ & $0.27(0.15$ to 0.47$)$ & $9.79(4.62$ to 20.7$)$ & $0.75(0.69$ to 0.81$)$ & $0.50(0.37$ to 0.62$)$ \\
\hline Recall difference place & 212 & $1.76(1.45$ to 2.13$)$ & $0.27(0.13$ to 0.55$)$ & $6.52(2.87$ to 15.0$)$ & $0.69(0.62$ to 0.75$)$ & $0.37(0.24$ to 0.49$)$ \\
\hline Forget where left things & 244 & $1.34(1.18$ to 1.52$)$ & $0.28(0.12$ to 0.66$)$ & $4.78(1.87$ to 12.2$)$ & $0.61(0.56$ to 0.67$)$ & $0.23(0.13$ to 0.33$)$ \\
\hline Any functioning problems & 217 & $1.48(1.27$ to 1.74$)$ & $0.28(0.13$ to 0.61$)$ & $5.28(2.18$ to 12.7$)$ & $0.64(0.59$ to 0.70$)$ & $0.39(0.24$ to 0.53$)$ \\
\hline Planning & 149 & $4.35(2.78$ to 6.83$)$ & $0.29(0.17$ to 0.49$)$ & $15.0(6.49$ to 34.8$)$ & $0.79(0.72$ to 0.87$)$ & $0.59(0.42$ to 0.71$)$ \\
\hline Recall where kept & 211 & $3.23(2.32$ to 4.50$)$ & $0.35(0.22$ to 0.55$)$ & $9.27(4.56$ to 18.9$)$ & $0.75(0.68$ to 0.82$)$ & $0.50(0.35$ to 0.63$)$
\end{tabular}

$A U C=$ area under receiver-operating characteristic $(R O C)$ curve. $D O R=$ diagnostic odds ratio. $L R-=$ negative likelihood ratio. $L R+=$ positive likelihood ratio. ${ }^{a}$ For details of clinical items see Appendix 1. 


\section{Table 4. Diagnostic utility of the best models}

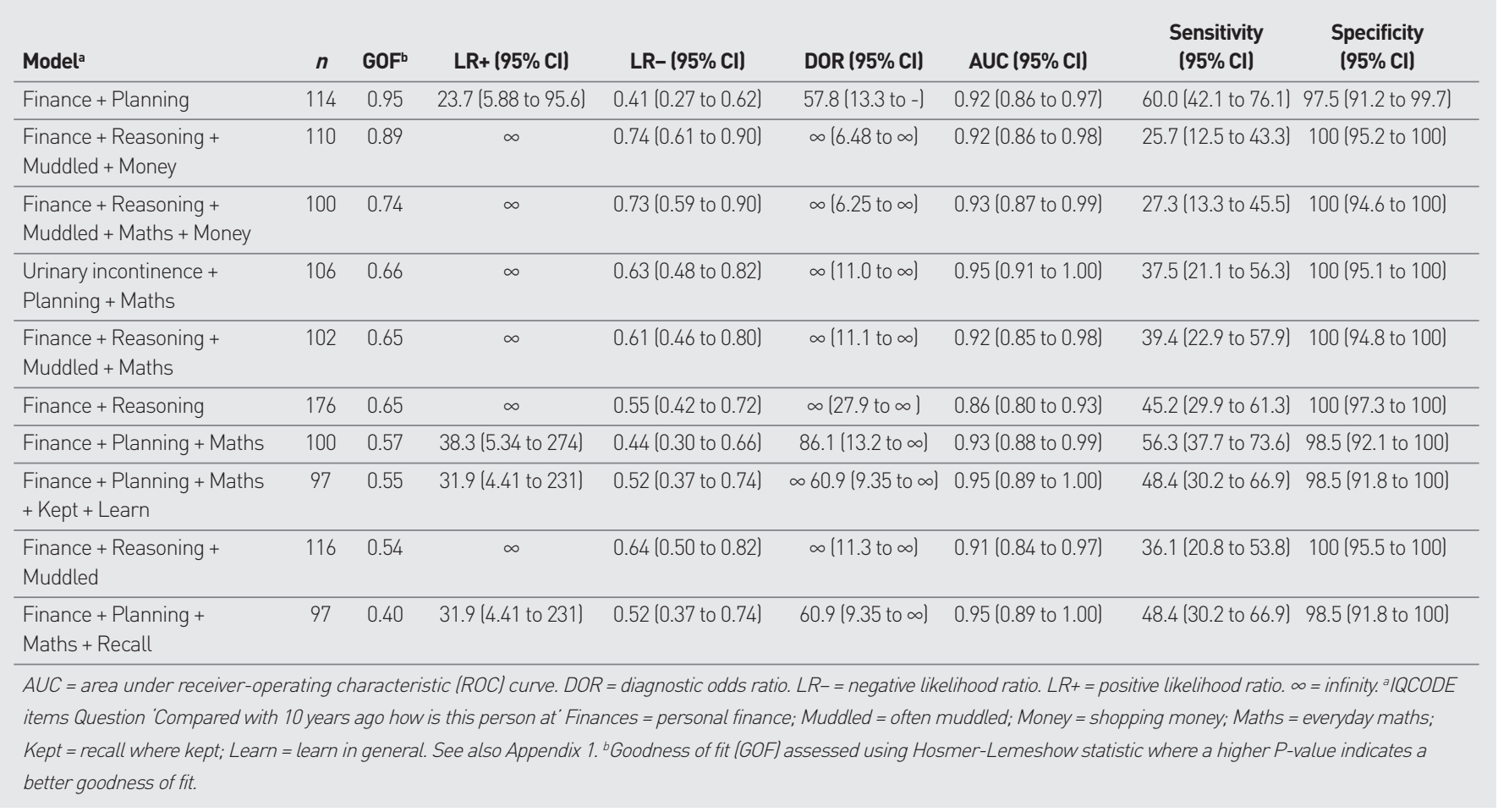

\section{Funding}

The main Caerphilly study was funded by an intramural grant to Medical Research Council Epidemiology Unit (Cardiff). The Alzheimer's Society funded the phase 5 follow-up. The sponsors had no role in the design and conduct of the study; collection, management, analysis, and interpretation of the data; and preparation, review, or approval of the manuscript.

\section{Ethical approval}

Ethical approval for phase 5 was provided by Gwent Research Ethics Committee (01/69).

\section{Provenance}

Freely submitted; externally peer reviewed.

\section{Competing interests}

The authors have declared no competing interests.

\section{Acknowledgements}

Professor Shah Ebrahim obtained funding for and helped design CaPS phase 5. We thank the reviewers and Professor Sarah Purdy for providing helpful comments that improved the manuscript. This study was presented at a UK academic general practice trainees conference, Oxford, April 2014.

\section{Discuss this article}

Contribute and read comments about this article: bjgp.org/letters of dementia. The estimates for diagnostic utility require validation in further studies, although the index test items were generally derived from well-validated instruments or standard clinical practice. When multipleimputation was used to deal with the issue of missing data, there was minimal change in the specificity, but the sensitivity of the clinical items was, in general, lower. One limitation is that the study findings cannot be generalised to women, and there may be sex-related and socially-determined patterns in the performance of some index tests. Although the authors examined for interactions, they were underpowered to detect them

The estimates of diagnostic utility may not generalise to other populations, with different prevalence of disease, as sampling was done on the basis of cognitive testing. The overall utility (AUC) of 'any memory difficulty' reported by either subject or informant was close to chance, suggesting that the sampling strategy oversampled men with cognitive impairment and hence would overestimate the PPV but would have underestimated the specificity. ${ }^{32}$ Other investigators have found that subjective memory problems are inconsistently associated with dementia. ${ }^{33}$ The estimates for diagnostic utility are likely to be overoptimistic as they result from stepwise selection procedures; some items may have been identified as being diagnostically useful by chance.

\section{Comparison with existing literature}

To the authors knowledge, this is the first study that has investigated the utility of such a broad range of clinical items for diagnosing dementia in a community-based sample. The results are supported by findings from other investigators who demonstrated that most diagnostic information in primary care was gained from age, functional assessment, ${ }^{34}$ and the clock drawing test (AUC 0.92). ${ }^{35}$ The diagnostic utility of an abnormal neurological examination ${ }^{36}$ and urinary incontinence ${ }^{37}$ were examined, which have been shown previously to be associated with dementia. Items on everyday maths, personal finances, and reasoning were all derived from IQCODE, ${ }^{26}$ and were previously found to have modest utility (AUC 0.85, 0.82, 0.82, respectively) in a case-control study in secondary care. ${ }^{38} \mathrm{As}$ a whole, the IQCODE is sensitive $(0.80)$ and specific (0.84) for diagnosing dementia, ${ }^{39}$ and functioning items have been found to be more discriminatory than memory items, ${ }^{40}$ which is in keeping with the present results.

\section{Implications for research and practice}

The current policy in the UK is to encourage GPs to diagnose dementia in typical elderly cases without referral ${ }^{11}$ unless there are 
specific reasons (for example, young age) that require a specialist opinion. Avoiding overdiagnosis (high specificity) may be more important than finding all possible cases (high sensitivity), as currently there are no drugs that modify the natural history of dementia ${ }^{41}$ and there are potential disadvantages from overdiagnosis. 111,42

In this study, simple questions had high post-test probability of unspecified dementia. In principle, this raises the possibility that GPs who are considering dementia after evaluating a person with symptoms might be able to make the diagnosis, without specialist input, using relatively simple adjunct questions. GPs who are being asked to diagnose dementia in primary care might find measures of functional performance more useful than standard tests of cognition.

These findings, if replicated in other settings, would potentially be of clinical value in the assessment of patients who are frail and older, a group in whom post-mortem studies indicate a weaker association between neuropathology and clinical presentation. ${ }^{7}$ It is not the intention that these questions should be used in isolation to diagnose dementia, but rather that they have added value after routine clinical assessment. This should not preclude the consideration of reversible causes of cognitive impairment ffor example, infection or depression), the use of further investigations to exclude pathologies (for example, space-occupying-lesion) that mimic dementia, or specialist referral for dementia subtype diagnosis. Such a two-pronged approach would enable GP diagnosis for some patients, with advanced work-up and referral being reserved for scenarios where diagnostic uncertainty persists. ${ }^{43}$ The utility of the observations in routine clinical settings requires further research. A prospective diagnostic test accuracy study is being conducted to validate these preliminary findings in men and women, and to quantify the incremental value of tests in the context of GP clinical suspicion of possible dementia. 


\section{REFERENCES}

1. Le Couteur DG, Doust J, Creasey H, Brayne C. Political drive to screen for predementia: not evidence based and ignores the harms of diagnosis. BMJ 2013; 347(sep09_21): f5125.

2. Burns A. Alistair Burns and 51 colleagues reply to David Le Couteur and colleagues. BMJ 2013; 347(oct15_6): $\{6125$.

3. Fox $C$, Lafortune $L$, Boustani $M$, Brayne $C$. The pros and cons of early diagnosis in dementia. Br J Gen Pract 2013; DOI: 10.3399/bjgp13X669374.

4. Ahmad S, Orrell M, Iliffe S, Gracie A. GPs' attitudes, awareness, and practice regarding early diagnosis of dementia. Br J Gen Pract 2010; DOI: 10.3399/ bjgp10X515386

5. Manthorpe J, Samsi K, Campbell S, et al. From forgetfulness to dementia: clinical and commissioning implications of diagnostic experiences. $\mathrm{Br} J \mathrm{Gen}$ Pract 2013; DOI: 10.3399/bjgp13X660805

6. Matthews FE, Brayne C, Lowe J, et al. Epidemiological pathology of dementia: attributable-risks at death in the Medical Research Council Cognitive Function and Ageing Study. PLoS Med 2009; 6(11): e1000180.

7. Neuropathology Group. Medical Research Council Cognitive Function and Aging Study. Pathological correlates of late-onset dementia in a multicentre community-based population in England and Wales. Neuropathology Group of the Medical Research Council Cognitive Function and Ageing Study (MRC CFAS). Lancet 2001; 357(9251): 169-175.

8. Sawa GM, Wharton SB, Ince PG, et al. Age, neuropathology, and dementia. N Engl J Med 2009; 360(22): 2302-2309

9. Dodd $\mathrm{E}$, Cheston $\mathrm{R}$, Fear $\mathrm{T}$, et al. An evaluation of primary care led dementia diagnostic services in Bristol. BMC Health Serv Res 2014; 14(1): 592

10. Greaves I, Greaves N, Walker E, et al. Gnosall Primary Care Memory Clinic: Eldercare facilitator role description and development. Dementia 2013; DOI: 10.1177/1471301213497737 [Epub ahead of print].

11. Brunet M. Targets for dementia diagnoses will lead to overdiagnosis. BMJ 2014 348(apr01_2): g2224.

12. National Institute for Health and Care Excellence. Support for commissioning dementia care NICE commissioning guides [CMG48] 5.1 Improving early identification, assessment and diagnosis. https://uww.nice.org.uk/guidance/ cmg48/chapter/51-improving-early-identification-assessment-and-diagnosis London: NICE, 2013

13. Dua T, Barbui C, Clark N, et al. Evidence-based guidelines for mental, neurological, and substance use disorders in low- and middle-income countries: summary of WHO recommendations. PLoS Med 2011; 8(11) e1001122.

14. Van Hout HPJ, Vernooij-Dassen MJFM, Hoefnagels WHL, et al. Dementia: predictors of diagnostic accuracy and the contribution of diagnostic recommendations. J Fam Pract 2002; 51(8): 693-699.

15. Pfeffer RI, Kurosaki TT, Harrah CH, et al. Measurement of functional activities in older adults in the community. J Gerontol 1982; 37(3): 323-329.

16. Folstein MF, Folstein SE, McHugh PR. "Mini-mental state". A practical method for grading the cognitive state of patients for the clinician. J Psychiatr Res 1975; 12(3): 189-198.

17. Cruz-Orduña I, Bellón JM, Torrero P, et al. Detecting $\mathrm{MCl}$ and dementia in primary care: effectiveness of the MMS, the FAQ and the IQCODE [corrected]. Fam Pract 2012: 29(4): 401-406.

18. McDowell I, Kristjansson B, Hill GBB, et al. Community screening for dementia: The Mini Mental State Exam (MMSEl and modified Mini-Mental State Exam (3MS) compared. J Clin Epidemiol 1997; 50(4): 377-383

19. Koch T, lliffe S. Rapid appraisal of barriers to the diagnosis and management of patients with dementia in primary care: a systematic review. BMC Fam Pract 2010; 11: 52.

20. Kroenke K, Spitzer RL, Williams JBW. The Patient Health Questionnaire-2: validity of a two-item depression screener. Med Care 2003; 41(11): 1284-1292.

21. The Caerphilly and Speedwell Collaborative Group. Caerphilly and Speedwell collaborative heart disease studies. The Caerphilly and Speedwell Collaborative Group. J Epidemiol Community Health 1984; 38(3): 259-262.

22. Fish M, Bayer A, Gallacher JEJ, et al. Prevalence and pattern of cognitive impairment in a community cohort of men in South Wales: methodology and findings from the Caerphilly Prospective Study. Neuroepidemiology 2008; 30(1): 25-33.

23. American Psychiatric Association. Diagnostic and Statistical Manual of Mental Disorders, Fourth Edition, Text Revision (DSM-IV-TR) [Internet]. Text. Washington, DC: American Psychiatric Association, 2000

24. Roth M, Tym E, Mountjoy CQ, et al. CAMDEX. A standardised instrument for the diagnosis of mental disorder in the elderly with special reference to the early detection of dementia. Br J Psychiatry 1986; 149: 698-709.

25. Dubois B, Slachevsky A, Litvan I, Pillon B. The FAB: a Frontal Assessment Battery at bedside. Neurology 2000; 55(11): 1621-1626.

26. Jorm AF, Korten AE. Assessment of cognitive decline in the elderly by informant interview. Br J Psychiatry 1988; 152: 209-213.

27. Meng X, D'Arcy C, Morgan D, Mousseau DD. Predicting the risk of dementia among Canadian seniors: a useable practice-friendly diagnostic algorithm. Alzheimer Dis Assoc Disord 2013; 27(1): 23-9.

28. Yin J, Tian L. Optimal linear combinations of multiple diagnostic biomarkers based on Youden index. Stat Med 2014; 33(8): 1426-1440.

29. Hosmer DW, Lemesbow S. Goodness of fit tests for the multiple logistic regression model. Commun Stat Methods 1980; 9(10): 1043-1069.

30. Van Buuren S, Boshuizen HC, Knook DL. Multiple imputation of missing blood pressure covariates in survival analysis. Stat Med 1999; 18(6): 681-694.

31. Worster A, Carpenter C. Incorporation bias in studies of diagnostic tests: how to avoid being biased about bias. CJEM 2008; 10(2): 174-175.

32. Leeflang MMG, Rutjes AWS, Reitsma JB, et al. Variation of a test's sensitivity and specificity with disease prevalence. CMAJ 2013; 185(11): E537-544.

33. Reid LM, Maclullich AMJ. Subjective memory complaints and cognitive impairment in older people. Dement Geriatr Cogn Disord 2006; 22(5-6): 471 485

34. Heyrman J, Dessers L, de Munter M-B, et al. Functional status assessment in the elderly. In: Wonca Classification Committee, ed. Functional status measurement in primary care. New York, NY: Springer, 1990: 213-221.

35. De Lepeleire J, Heyrman J, Baro F, Buntinx F. A combination of tests for the diagnosis of dementia had a significant diagnostic value. J Clin Epidemiol 2005; 58(3): 217-225

36. Olazarán J, Torrero P, Cruz I, et al. Mild cognitive impairment and dementia in primary care: the value of medical history. Fam Pract 2011; 28(4): 385-392.

37. Grant RL, Drennan VM, Rait G, et al. First diagnosis and management of incontinence in older people with and without dementia in primary care: a cohort study using The Health Improvement Network primary care database. PLoS Med 2013; 10(8): e1001505.

38. Perroco TR, Bustamante SEZ, Moreno MDPQ et al. Performance of Brazilian long and short IQCODE on the screening of dementia in elderly people with low education. Int Psychogeriatr 2009; 21(3): 531-538.

39. Quinn TJ, Fearon P, Noel-Storr AH, et al. Informant Questionnaire on Cognitive Decline in the Elderly (IQCODE) for the diagnosis of dementia within community dwelling populations. Cochrane Database Syst Rev 2014; 4: CD010079.

40. Sikkes SAM, Knol DL, van den Berg MT, et al. An informant questionnaire for detecting Alzheimer's disease: are some items better than others? J Int Neuropsychol Soc 2011; 17(4): 674-681.

41. Gordon PJ. No evidence exists that 'anti-dementia' drugs modify disease or improve outcome. BMJ 2014; 348: g2607.

42. Brunet MD, McCartney M, Heath I, et al. There is no evidence base for proposed dementia screening. BMJ 2012; 345: e8588.

43. Health Quality Ontario. The appropriate use of neuroimaging in the diagnostic work-up of dementia: an evidence-based analysis. Ont Health Technol Assess Ser 2014; 14(1): 1-64. 


\section{Appendix 1. Details of items in index test evaluation}

\begin{tabular}{|c|c|}
\hline Item (full details) & Referred to in manuscript as \\
\hline \multicolumn{2}{|l|}{ Initial questions: asked of both subject and informant } \\
\hline Have any difficulty with your memory? & Any memory difficulty \\
\hline Forget where you left things more than you used to? & Forget where left things \\
\hline Forget what you were going to do, on the way to doing it? & Forget what going to do \\
\hline Forget names of close friends or relatives? & Forget names \\
\hline Ever forgotten your way or got lost in own neighbourhood? & Get lost \\
\hline Forget what said \& ask same question over \& over? & Repeat question \\
\hline Aware of memory problem & Aware of problem \\
\hline Difficulty finding word you want to say? & Word finding difficulty \\
\hline Sometimes say the wrong word? & Wrong word \\
\hline \multicolumn{2}{|l|}{ Interviewer observations } \\
\hline Self-neglect & Interviewer: Self-neglect \\
\hline Uncooperative behaviour & Interviewer: Uncooperative \\
\hline Suspiciousness & Interviewer: Suspiciousness ${ }^{a}$ \\
\hline Hostile/irritable & Interviewer: Hostile \\
\hline Incongruent/bizarre & Interviewer: Bizarre \\
\hline Slow/underactive & Interviewer: Slow \\
\hline Restless & Interviewer: Restless \\
\hline Anxious out of proportion to situation & Interviewer: Anxious \\
\hline Looks depressed & Interviewer: depressed \\
\hline Emotional lability & Interviewer: Labile \\
\hline Flat affect & Interviewer: Flat \\
\hline Appears to be hallucinating & Interviewer: Hallucinating ${ }^{\mathrm{a}}$ \\
\hline Rapid speech & Interviewer: Rapid speech \\
\hline Slow speech & Interviewer: Slow speech \\
\hline No spontaneous speech, restricted in quantity & Interviewer: limited speech \\
\hline Speech rambling, incoherent, irrelevant & Interviewer: Speech rambling \\
\hline Speech slurred & Interviewer: Speech slurred \\
\hline Perseverating & Interviewer: Perseveratinga \\
\hline No insight & Interviewer: No insight \\
\hline Clouding of consciousness & $\begin{array}{l}\text { Interviewer: Clouding of } \\
\text { consciousness }\end{array}$ \\
\hline Peculiar use of terms & Interviewer: Peculiar terms \\
\hline Speaking to self & Interviewer: Speaking to self \\
\hline Impaired ability to focus, shift or sustain attention & Interviewer: Impaired focus \\
\hline Impaired judgement of situations or persons & Interviewer: Impaired judgement \\
\hline Hypochondriacal preoccupations with somatic discomfort & Interviewer: Hypochondriacala \\
\hline Repetitive conversation & Interviewer: Repetitive \\
\hline Spontaneously talking about distant past & Interviewer: Distant past \\
\hline \multicolumn{2}{|l|}{ Examination Findings } \\
\hline Other significant findings & Other significant findings \\
\hline Cranial Nerve abnormality & Cranial nerve defect \\
\hline Field defect & Field defect \\
\hline Horner Syndrome & Horner's \\
\hline Acuity & Acuity \\
\hline Ophthalmoplegia & Ophthalmoplegia \\
\hline VII cranial nerve deficit & Facial nerve palsy \\
\hline Hearing difficulty & Hearing difficulty \\
\hline
\end{tabular}




\section{Appendix 1 continued. Details of items in index test evaluation}

\begin{tabular}{|c|c|}
\hline Pseudo-bulbar palsy & Pseudo-bulbar palsy \\
\hline Bulbar palsy & Bulbar palsy \\
\hline Speech disturbance: Dysarthria & Dysarthria \\
\hline Gait disturbance any & Any Gait disturbance \\
\hline Gait disturbance: Parkinsonian/Apraxic & Parkinsonian gait \\
\hline Gait disturbance: Spastic & Spastic gait \\
\hline Gait disturbance: Ataxic & Ataxic gait \\
\hline Gait disturbance: Antalgic & Antalgic gait \\
\hline $\begin{array}{l}\text { Pyramidal Signs (not clinically due to myelopathy): } \\
\text { on right, left or absent }\end{array}$ & Significant pyramidal signs \\
\hline Drift & Drift \\
\hline Weakness & Weakness \\
\hline Hypertonia & Hypertonia \\
\hline Hyperreflexia & Hyperreflexia \\
\hline Babinski & Babinski \\
\hline Extrapyramidal Signs & Significant Extrapyramidal Signs \\
\hline Rigidity & Rigidity \\
\hline Bradykinesia & Bradykinesia \\
\hline Tremor & Tremor \\
\hline Postural instability & Postural instability \\
\hline Asymmetric deficit & Asymmetric deficit \\
\hline Central Sensory disturbance (not peripheral nerve) & Sensory disturbance \\
\hline Cerebellar signs & Cerebellar signs \\
\hline Other localising signs & Other localising signs \\
\hline Primitive reflexes & Any primitive reflexes \\
\hline Palmomental & Palmomental \\
\hline Pout & Pout \\
\hline Grasp & Grasp \\
\hline \multicolumn{2}{|l|}{ IQCode. Compared with 10 years ago how is this person at: } \\
\hline $\begin{array}{l}\text { Remembering things about family and friends } \\
\text { (for example, birthdays, occupations, addresses) }\end{array}$ & Family and friends \\
\hline Remembering things that have happened recently & Recall recent events \\
\hline Recalling conversations a few days later & Recall conversations \\
\hline Remembering own address and telephone number & Recall own address \\
\hline Remembering what day and month it is & Recall day \\
\hline Remembering where things are usually kept & Recall where kept \\
\hline $\begin{array}{l}\text { Remembering where to find things put in a different } \\
\text { place from usual }\end{array}$ & Recall difference place \\
\hline Working familiar household appliances & Work appliances \\
\hline Learning to use new gadget or appliance around the house & Learn new gadget \\
\hline Learning new things in general & Learn in general \\
\hline Following a story in a book or on TV & Follow story \\
\hline Making decisions on everyday matters & Decisions \\
\hline Handling money for shopping & Shopping money \\
\hline Handling personal finance (for example, pension, bank) & Personal finance \\
\hline $\begin{array}{l}\text { Handling everyday arithmetic (for example, how much food } \\
\text { to buy, time between visits from friends/family }\end{array}$ & Everyday maths \\
\hline $\begin{array}{l}\text { Using intelligence to understand what's going on and } \\
\text { to reason things through }\end{array}$ & Reasoning \\
\hline
\end{tabular}




\section{Appendix 1 continued. Details of items in index test evaluation}

Everyday Activities (asked of both subject and informant)

$\begin{array}{ll}\text { Any problems with daily activities } & \text { Any ADL problems } \\ \text { Difficulty feeding self } & \text { Feeding } \\ \text { Change in eating habit } & \text { Appetite } \\ \text { Difficulty dressing } & \text { Dressing } \\ \text { Needing assistance with bathing/washing/toileting } & \text { Toileting } \\ \text { Decreased attention to personal hygiene } & \text { Hygiene }\end{array}$

\section{Physical Symptoms (informant)}

Physical symptoms (any)

Any physical symptoms

Urinary

Urinary incontinence

Faecal

Faecal incontinence

Tendency to fall

Falls

Syncope/unexplained loss of consciousness

Syncope

\section{General Functioning report (informant)}

General functioning any problems

Any functioning problems

Tends to talk about distant past rather than present

Distant past

Loss of special skill or hobby

Thinking often muddled

Hobby

Greater difficulty thinking and planning ahead

Often muddled

Planning

Difficulty concentrating, more distractible

Concentrating

Talking more than previously

Talk more

Talking less than previously

Talk less

Repeating words or phrases

Repeat words

Fails to realise extent of any problems

No insight

Personality/emotion/behaviour (informant)
Any personality change
Any personality change
Emotional lability
Labile
Emotional blunting
Blunting
Lack of motivation and spontaneous behaviour
Unmotivated
Breaches of social etiquette/disinhibited behaviour
Disinhibited
Perservative/stereotyped behaviour
Perseveration
Sleep pattern disturbance
Sleep disturbed
Aggression
Aggressive
Narrowed preoccupations
Mental rigidity
Hyperorality (increased oral behaviours)
Narrowed preoccupations
Mental rigidity
Auditory hallucinations
Hyperorality
Visual hallucinations
Auditory hallucinations
Visual hallucinations
Would (Has) subject cope (d) alone without Unable cope alone partner/cohabitee?

\section{Symptom onset and progression}

Onset Months since onset: more than 12

Course of symptoms

Course not improving

\section{Symptom fluctuation}

Symptom fluctuation (any)

Hour to hour

Hour to hour

Day to night

Day to night

Day to day

Day to day

Week to week

Week to week

Accommodation not own home

Accommodation not own home

alndicates features which were not identified in any men and so diagnostic utility could not be calculated. $A D L=$ activities of daily living. 